BULL. AUSTRAL. MATH. SOC.

VOL. 31 (1985), 199-2I4.

\title{
LEBESGUE CONSTANTS FOR DOUBLE HAUSDORFF MEANS
}

\author{
F. USTINA
}

As is well known, the divergence of the set of constants known as the Lebesgue constants corresponding to a particular method of summability implies the existence of a continuous, periodic function whose Fourier series, summed by the method, diverges at a point, and of another such function the sums of whose Fourier series converge everywhere but not uniformly in the neighborhood of some point.

In 1961, Lorch and Newman established that if $L(n ; g)$ is the $n$th Lebesgue constant for the Hausdorff summability method corresponding to the weight function $g(u)$, then

$$
L(n ; g)=C(g) \log n+o(\log n), n \rightarrow \infty,
$$

where

$$
C(g)=\left(2 / \pi^{2}\right)\left|g(1)-g\left(1^{-}\right)\right|+(1 / \pi) M\left\{\left|\sum\left\{g\left(\varepsilon_{k}^{+}\right)-g\left(\varepsilon_{k}^{-}\right)\right\} \sin \varepsilon_{k} u\right|\right\},
$$

where the summation is taken over the jump discontinuities $\left\{\varepsilon_{k}\right\}$

of $g(u)$ and $M\{f(u)\}$ denotes the mean value of the almost

periodic function $f(u)$.

In this paper, a partial extension of this result to the two dimensional analogue is obtained. This extension is summarized in Theorem 1.3.

Received 5 September 1984.

Copyright Clearance Centre, Inc. Serial-fee code: 0004-9727/85 $\$$ A2.00 +0.00 . 


\section{Introduction}

The Lebesgue constants for double Fourier series are defined by

$$
L(m, n)=4 / \pi^{2} \int_{0,0}^{\pi, \pi}\left|D_{m, n}(s, t)\right| d s d t
$$

where

$$
D_{m, n}(s, t)=\frac{\sin \left(m+\frac{1}{2}\right) s}{2 \sin \frac{1}{2} s} \frac{\sin \left(n+\frac{1}{2}\right) t}{2 \sin \frac{1}{2} t}
$$

is the Dirichlet kernel. As is well known, the divergence of these constants implies the existence of a continuous, periodic function of two variables whose Fourier series diverges at a point (the du Bois-Reymond singularity), and another such function whose Fourier series converges everywhere, but not uniformly in the neighborhood of some point (the Lebesgue singularity).

If the sequence $\left\{D_{k, Z}(s, t)\right\}$ is transformed by a summability method $S$, and we denote the $m n$th transform of this sequence by $K_{m, n}(s, t)$, then the sequence of constants defined by

$$
L(m, n ; s)=4 / \pi^{2} \int_{0,0}^{\pi, \pi}\left|K_{m, n}(s, t)\right| d s d t
$$

are the Lebesgue constants for the summability method $S$. The divergence of these constants implies the du Bois-Reymond singularity and the Lebesgue singularity for the summability method $S$.

Lorch and Newman [4] investigated the Lebesgue constants for the regular Hausdorff means in the one dimensional case and established the following :

THEOREM 1.2. Let $L(m ; g)$ denote the $m$ th Lebesgue constant for the regular Hausdorff method with weight function $g(u)$. Then

$$
L(m ; g)=c(g) \log m+o(\log m), m+\infty,
$$

where, with $u_{k}$ the $k$ th discontinuity of $g(u)$ and $M\{f(x)\}$ the mean value of the almost periodic function $f(x)$,

$$
c(g)=\frac{2}{\pi^{2}}\left|g(1)-g\left(I^{-}\right)\right|+\frac{1}{\pi} M\left\{\left|\sum_{k}\left\{g\left(u_{k}^{+}\right)-g\left(u_{k}^{-}\right)\right\} \sin u_{k} x\right|\right\} \text {. }
$$


Furthermore,

$$
0 \leq c(g) \leq\left(4 / \pi^{2}\right) v(g), \quad 0 \leq u \leq 1,
$$

and $c(g)=0$ if and only if $g(u)$ is continuous. If the method is totally regular so that $V(g)=1$, then $c(g)=4 / \pi^{2}$ if and only if the method is ordinary convergence. If $g(1)=g\left(1^{-}\right)$, then $c(g) \leq 2 / \pi^{2}$, and $c(g)=2 / \pi^{2}$ if and only if the method is of Euler type.

The object of this paper is to get a partial extension of the above result to the two dimensional case. A full extension will follow in a subsequent paper. More specifically, we prove

THEOREM 1.3. If $L(m, n ; g)$ is the mnth Lebesgue constant for the Hausdorff means corresponding to the regular weight function $g(u, v)$, then

$$
\begin{aligned}
L(m, n ; g)= & 4 / \pi^{2} \int_{1,1}^{n^{\frac{1}{2}}, m^{\frac{1}{2}}}\left|\int_{0,0}^{1,1} \frac{\operatorname{sinsu}}{s} \frac{\sin t v}{t} d g(u, v)\right| d s d t \\
& +4 / \pi^{3} \log n \int_{1}^{m^{\frac{1}{2}}}\left|\int_{0}^{1} \frac{\operatorname{sinsu}}{s}\left\{d g(u, 1)-d g\left(u, 1^{-}\right)\right\}\right| d s \\
& +4 / \pi^{3} \log m \int_{1}^{n^{\frac{1}{2}}}\left|\int_{0}^{1} \frac{\sin t v}{t}\left\{d g(1, v)-d g\left(1^{-}, v\right)\right\}\right| d t \\
& +4 / \pi^{4} \lg \left(1,1 ; 1^{-}, I^{-}\right) \mid \log m \log n+o(\log m \log n),
\end{aligned}
$$

as $m, n \rightarrow \infty$, where

$$
g\left(1,1 ; 1^{-}, 1^{-}\right)=g(1,1)-g\left(1,1^{-}\right)-g\left(1^{-}, 1\right)+g\left(1^{-}, 1^{-}\right) \text {. }
$$

If $g(u, 1)=g\left(u, 1^{-}\right)$and $g(1, v)=g\left(1^{-}, v\right), 0 \leq u, v<1$, then

(1.5) $L(m, n ; g)=4 / \pi^{2} \int_{1,1}^{n^{\frac{1}{2}}, m^{\frac{1}{2}}}\left|\int_{0,0}^{1,1} \frac{\text { sinsu }}{s} \frac{\text { sint } t v}{t} d g(u, v)\right| d s d t$

$$
+4 / \pi^{4} \lg \left(1,1 ; 1^{-}, 1^{-}\right) \mid \log m \log n+o(\log m \log n) \text {, }
$$

as $m, n \rightarrow \infty$. If, in addition, $g(u, v)$ is continuous in its domain at $(1,1)$, or if only $g\left(1,1 ; 1^{-}, 1^{-}\right)=0$, then 
(1.6) $L(m, n ; g)=4 / \pi^{2} \int_{1,1}^{n^{\frac{1}{2}}, m^{\frac{1}{2}}}\left|\int_{0,0}^{1,1} \frac{\text { sinsu }}{s} \frac{\sin t v}{t} d g(u, v)\right| d s d t$ $+o(\log m \log n), m, n \rightarrow \infty$.

NOTE 1.7. In the sequel, all $O_{-}$and $O_{-}$terms will be taken as $m$ and $n$ tend to $\infty$ without further mention.

\section{A preliminary result}

If $K_{m, n}(s, t)$ is the mnth Hausdorff transform of the sequence $\left\{D_{k, Z}(s, t)\right\}$, corresponding to the regular weight function $g(u, v)$, then (2.1) $K_{m, n}(s, t)$

$$
\begin{aligned}
& =\sum_{k, l=0}^{m, n}\left(\begin{array}{l}
n \\
l
\end{array}\right)\left(\begin{array}{l}
m \\
k
\end{array}\right) D_{k, l}(s, t) \int_{0,0}^{1,1} u^{i}(1-u)^{m-k} v^{l}(1-v)^{n-l} d g(u, v) \\
& =\frac{3}{4} \int_{0,0}^{1,1} \frac{\operatorname{Im}\left\{\left(1-u+u e^{i s}\right)^{m} e^{i(s / 2)}\right.}{\sin \frac{1}{2} s} \frac{\operatorname{Im}\left\{\left(1-v+v e^{i t}\right)^{n} e^{i(t / 2)}\right\}}{\sin \frac{1}{2} t} d g(u, v) \\
& =\frac{1}{4} \int_{0,0}^{1,1} \rho_{1}^{m} \rho_{2}^{n} \sin m \alpha \sin n \beta \cot \frac{s}{2} \cot \frac{t}{2} d g(u, v) \\
& +\frac{1}{4} \int_{0,0}^{1,1} \rho_{1}^{m} \rho_{2}^{n} \sin m \alpha \cos n \beta \cot \frac{s}{2} d g(u, v) \\
& +\frac{1}{4} \int_{0,0}^{1,1} \rho_{1}^{m} \rho_{2}^{n} \sin n \beta \cos m \alpha \cot \frac{t}{2} d g(u, v) \\
& +\frac{1}{4} \int_{0,0}^{1,1} \rho_{1}^{m} \rho_{2}^{n} \cos m \alpha \cos n \beta d g(u, v)
\end{aligned}
$$

where $\rho_{1}, \rho_{2}, \alpha$ and $B$ are defined as in [6] by

$$
\begin{aligned}
& \rho_{1} \sin \alpha=u \sin s, \rho_{1} \cos \alpha=1-u+u \cos s \\
& \rho_{2} \sin \beta=v \sin t, \rho_{2} \cos \beta=1-v+v \cos t
\end{aligned}
$$

or

$$
\rho_{1} e^{i \alpha}=1-u+u e^{i s}, \rho_{2} e^{i \beta}=1-v+v e^{i t}
$$

It follows that 
(2.4) $\rho_{1}^{2}=1-2 u(1-u)(1-\cos s), \rho_{2}^{2}=1-2 v(1-v)(1-\cos t)$. Hence $0 \leq \rho_{1}^{2}, \rho_{2}^{2} \leq 1$ in the region of integration.

Substituting $\cot x / 2=\{\cot x / 2-2 / x\}+2 / x$ in $(2.1)$ and simplifying the results it follows that

(2.5) $K_{m, n}(s, t)=\int_{0,0}^{1,1} \rho_{1}^{m} \rho_{2}^{n} \frac{\sin m \alpha}{s} \frac{\sin n \beta}{t} d g(u, v)$

$$
\begin{aligned}
& +\int_{0,0}^{1,1} \rho_{1}^{m} \frac{\sin m \alpha}{s} \phi(n ; t, v) d g(u, v) \\
& +\int_{0,0}^{1,1} \rho_{2}^{n} \frac{\sin n B}{t} \phi(m ; s, u) d g(u, v) \\
& +\int_{0,0}^{1,1} \phi(m ; s, u) \phi(n ; t, v) d g(u, v)
\end{aligned}
$$

where

$$
\phi(m ; s, u)=\rho_{1}^{m}\left\{\sin m \alpha\left\{\cot \frac{s}{2}-\frac{2}{s}\right\}+\cos m \alpha\right\}
$$

with $\phi(n ; t, v)$ defined in an analogous manner.

Note that by a lemma due to Livingston [3],

$$
\rho_{1}^{2}<e^{-2 u(1-u) s^{2} / \pi^{2}}
$$

Hence $\phi(m ; s, u)=o(1), m \rightarrow \infty$ and $\phi(n ; t, v)=o(1), n \rightarrow \infty$, $0<u, v<1$ and $0<s, t<\pi$.

Next, by a lemma due to Szász [5], $\alpha=\alpha(s, u)=s u+o\left(u(1-u) s^{3}\right)$. Hence

$$
\begin{aligned}
\left(\rho_{l}^{m} / s\right)(\sin m \alpha-\sin m s u) & =\left(2 \rho_{1}^{m} / s\right)\left(\cos \frac{m}{2}(\alpha+s u) \sin \frac{m}{2}(\alpha-s u)\right) \\
& =\left(2 \rho_{1}^{m} / s\right) \sin o\left(m u(1-u) s^{3}\right) \\
& <2 e^{-m u(1-u) s^{2} / \pi^{2}} o\left(m u(1-u) s^{2}\right) \\
& =o(1),
\end{aligned}
$$

uniformly in $s$ and $u$. Hence we may write 


$$
\begin{aligned}
& \rho_{1}^{m} \frac{\sin m \alpha}{s}=\rho_{1}^{m} \frac{\sin m s u}{s}+\psi(m ; s, u), \\
& \rho_{2}^{n} \frac{\sin n \beta}{t}=\rho_{2}^{n} \frac{\sin n t v}{t}+\psi(n ; t, v),
\end{aligned}
$$

where the $\phi$-function tends to zero. Substituting these in (2.5) and simplifying, it follows that

(2.8) $K_{m, n}(s, t)=\int_{0,0}^{1,1} \rho_{1}^{m} \rho_{2}^{n} \frac{\operatorname{sinmsu}}{s} \frac{\sin n t v}{t} d g(u, v)$

$$
\begin{aligned}
& +\int_{0,0}^{1, I} \rho_{1}^{m} \frac{\sin m s u}{s} \gamma(n ; t, v) d g(u, v) \\
& +\int_{0,0}^{1, I} \rho_{2}^{n} \frac{\sin n t v}{t} \gamma(n ; t, v) d g(u, v) \\
& +\int_{0,0}^{1, I} \gamma(m ; s, u) \gamma(n ; t, v) d g(u, v)
\end{aligned}
$$

where

$$
\begin{aligned}
& \gamma(m ; s, u)=\psi(m ; s, u)+\psi(m ; s, u), \\
& \gamma(n ; t, v)=\psi(n ; t, v)+\psi(n ; t, v) .
\end{aligned}
$$

Thus

$$
\text { (2.9) } \begin{aligned}
L(m, n ; g)=4 / \pi^{2} & \int_{0,0}^{\pi, \pi}\left|\int_{0,0}^{1,1} \rho_{1}^{m} \rho_{2}^{n} \frac{\operatorname{sinmsu}}{s} \frac{\sin n t v}{t} d g(u, v)\right| d s d t \\
& +o\left(\int_{0,0}^{\pi, \pi}\left|\int_{0,0}^{1,1} \rho_{1}^{m} \frac{\operatorname{sinmsu}}{2} \gamma(n ; t, v) d g(u, v)\right| d s d t\right) \\
& +o\left(\int_{0,0}^{\pi, \pi}\left|\int_{0,0}^{1,1} \rho_{2}^{n} \frac{\sin n t v}{2} \gamma(m ; s, u) d g(u, v)\right| d s d t\right) \\
& +o\left(\int_{0,0}^{\pi, \pi}\left|\int_{0,0}^{1,1} \gamma(m ; s, u) \gamma(n ; t, v) d g(u, v)\right| d s d t\right) .
\end{aligned}
$$

The last integral in (2.9) is clearly $O(1)$ or $O(\log m \log n)$. That the second and third integrals are also $o(\log m \log n)$ follows from the following.

LEMMA 2.10. If $h(m, n ; s, t, u, v)$ is uniformly bounded in each argument, and if $g(u, v)$ is of bounded variation in the sense of Hardy 
and Krause, then

$$
\begin{aligned}
& \int_{0,0}^{\pi, \pi}\left|\int_{0,0}^{1,1} h(m, n ; s, t, u, s) \frac{\sin m s u}{s} d g(u, v)\right| d s d t=o(\log m \log n) . \\
& \text { Proof. } \\
& \int_{0,0}^{\pi, \pi}\left|\int_{0,0}^{1,1} h(m, n ; s, t, u, v) \frac{\sin m s u}{s} d g(u, v)\right| d s d t \\
& \leq \int_{0,0}^{\pi, \pi} \int_{0,0}^{1,1}|h(m, n ; s, t, u, v)|\left|\frac{\operatorname{sinmsu}}{s}\right||d g(u, v)| d s d t \\
& =0(1) \int_{0}^{\pi} \int_{0,0}^{1,1}\left|\frac{\sin m s u}{s}\right||d g(u, v)| d s \\
& =O(1)\left\{\int_{0}^{1}+\int_{1}^{\pi m}\right\} \int_{0,0}^{1,1}\left|\frac{\sin s u}{s}\right||d g(u, v)| d s \\
& =O(1)\{1+\log \pi+\log m\} V(g) \\
& =O(\log m)=o(\log m \log n) \text {. }
\end{aligned}
$$

Thus the second and third integrals in (2.9) are also $o(\log m \log n)$. We state the result in a theorem.

THEOREM 2.11. If $L(m, n ; g)$ denotes the mnth Lebesgue constant for the Hausdorff means corresponding to the regular weight function $g(u, v)$, then

$$
\begin{array}{r}
L(m, n ; g)=\frac{4}{\pi^{2}} \int_{0,0}^{\pi, \pi}\left|\int_{0,0}^{1,1} \rho_{1}^{m} \rho_{2}^{n} \frac{\operatorname{sinmsu}}{s} \frac{\sin n t v}{t} d g(u, v)\right| d s d t \\
\quad+o(\log m \log n), m, n \rightarrow \infty,
\end{array}
$$

where $\rho_{1}^{2}=1+2 u(1-u)(1-\cos s)$ and $\rho_{2}^{2}=1-2 v(1-v)(1-\cos t)$.

\section{Proof of Theorem 1.3}

For $a<c$ and $b<d$, let $[c, d ; a, b]$ denote the rectangular cell with vertices at $(a, b),(a, d),(c, d)$ and $(c, b)$, and let $g(c, d ; a, b)=g(c, d)-g(a, d)-g(c, b)+g(a, b)$. Thus the integral in Theorem 2.11 is over the cell $[\pi, \pi ; 0,0]$ with respect to $s$ and $t$. Divide this cell into four subcells, $\left[m^{-\frac{1}{2}}, n^{-\frac{2}{2}} ; 0,0\right]$, $\left[m^{-\frac{1}{2}}, \pi ; 0, n^{-\frac{1}{2}}\right],\left[\pi, n^{-\frac{1}{2}} ; m^{-\frac{1}{2}}, 0\right]$ and $\left[\pi, \pi ; m^{-\frac{1}{2}}, n^{-\frac{1}{2}}\right]$, and denote 
this integral over the respective subcells by $I_{1}, I_{2}, I_{3}$ and $I_{4}$.

LEMMA 3.1 .

$$
I_{1}=\int_{1,1}^{n^{\frac{1}{2}}, m^{\frac{1}{2}}}\left|\int_{0,0}^{1,1} \frac{\operatorname{sinsu}}{s} \frac{\sin t v}{t} d g(u, v)\right| d s d t+o(\log m \log n) .
$$

Proof. By $(2.4)$,

$$
\begin{aligned}
\rho_{1}^{2} & =1-2 u(1-u)(1-\cos s) \\
& =1-4 u(1-u) \sin ^{2}(s / 2) \\
& \geq \cos ^{2}(s / 2) .
\end{aligned}
$$

Thus in the cell $\left[m^{-\frac{1}{2}}, n^{-\frac{1}{2}} ; 0,0\right], 1-m(s / 2)^{2} \leq \cos ^{m}(s / 2) \leq \rho_{1}^{m} \leq 1$.

Set $\rho_{1}^{m}=1+\phi_{m}(s, u)$ where $\left|\phi_{m}(s, u)\right| \leq m(s / 2)^{2}$. Similarly,

$\rho_{2}^{n}=1+\phi_{n}(t, v)$ where $\left|\phi_{n}(t, v)\right| \leq n(t / 2)^{2}$. Replacing $\rho_{1}^{m}$ and $\rho_{2}^{n}$

in the integral in (2.12) by these equivalents, it follows that

$$
\begin{aligned}
I_{1}= & \int_{0,0}^{n^{-\frac{1}{2}}, m^{-\frac{1}{2}}}\left|\int_{0,0}^{1,1} \frac{\sin m s u}{s} \frac{\sin n t v}{t} d g(u, v)\right| d s d t+o(1) \int_{0,0}^{n^{-\frac{1}{2}}, m^{-\frac{1}{2}}} \\
& \left|\int_{0,0}^{1,1}\left\{\phi_{m}(s, u)+\phi_{n}(t, v)+\phi_{m}(s, u) \phi_{n}(t, v)\right\} \frac{\sin m s u}{s} \frac{\sin n t v}{t} d t(u, v)\right| d s d t . \\
& \text { Now }\left|\phi_{m}(s, u)(\sin m s u) / s\right| \leq\left|\phi_{m}(s, u) / s\right| \leq m s / 4 \text {. Hence }
\end{aligned}
$$


(3.3) $\int_{0,0}^{n^{-\frac{1}{2}}, m^{-\frac{1}{2}}}\left|\int_{0,0}^{1,1} \phi_{m}(s, u) \frac{\sin m s u}{s} \frac{\sin n t v}{t} d g(u, v)\right| d s d t$

$$
\begin{aligned}
& \leq \int_{0,0}^{n^{-\frac{1}{2}}, m^{-\frac{1}{2}}}(m s / 4) \int_{0,0}^{1,1}\left|\frac{\sin n t v}{t}\right||d g(u, v)| d s d t \\
& =(1 / 8)\left\{\int_{0}^{1}+\int_{1}^{n^{\frac{1}{2}}}\right\} \int_{0,0}^{1,1}\left|\frac{\sin t v}{t}\right||d g(u, v)| d t \\
& <(1 / 8)\left\{1+\int_{1}^{n^{\frac{1}{2}}} \frac{d t}{t}\right\} \int_{0,0}^{1,1}|d g(u, v)| \\
& =(1 / 8)\left\{I+\frac{1}{2} \log n\right\} V(g) \\
& =0(\log m \log n),
\end{aligned}
$$

since $|(\sin t v) / t| \leq|(\sin t) / t| \leq 1$ in $(0,1]$ and $|(\sin t v) / t| \leq(1 / t), 1 \leq t$. Similarly,

(3.4) $\int_{0,0}^{n^{-\frac{1}{2}}, m^{-\frac{1}{2}}}\left|\int_{0,0}^{1,1} \phi_{n}(t, v) \frac{\sin m s u}{s} \frac{\sin n t v}{t} d g(u, v)\right| d s d t=o(\log m \log n)$ and

(3.5) $\int_{0,0}^{n^{-\frac{1}{2}}, m^{-\frac{1}{2}}}\left|\int_{0,0}^{1,1} \phi_{m}(s, u) \phi_{n}(t, v) \frac{\sin m s u}{s} \frac{\sin n t v}{t} d g(u, v)\right| d s d t$ $=o(1)=1(\log m \log n)$.

Thus by (3.3), (3.4) and (3.5), the last integral on the right in (3.2) is $o(\log m \log n)$.

Next, replace $m s$ by $s$ and $n t$ by $t$ in the remaining integral in (3.2) and obtain

(3.6) $I_{1}=\int_{0,0}^{n^{\frac{1}{2}}, m^{\frac{1}{2}}}\left|\int_{0,0}^{1,1} \frac{\text { sinsu }}{s} \frac{\sin t v}{t} d g(u, v)\right| d s d t+o(\log m \log n)$.

Finally, shifting the lower limit on the outer integral in (3.6) from $(0,0)$ to $(1,1)$ introduces an error of

$$
\left\{\int_{0,0}^{1,1}+\int_{0,1}^{1, m^{\frac{1}{2}}}+\int_{1,0}^{n^{\frac{1}{2}}, 1}\right\}\left|\int_{0,0}^{1,1} \frac{\operatorname{sinsu}}{s} \frac{\sin t v}{t} d g(u, v)\right| d s d t .
$$


The first integral is clearly $O(1)$ since $(\sin s u) / s$ and $(\sin t v) / t$ are bounded in the region of integration. The second and third integrals are $O(\log m)$ and $O(\log n)$ respectively, and their sum is $o(\log m \log n)$. Collecting the results, it follows that

$$
I_{1}=\int_{1, I}^{m^{\frac{1}{2}}, m^{\frac{3}{2}}}\left|\int_{0,0}^{1, I} \frac{\operatorname{sinsu}}{s} \frac{\sin t v}{t} d g(u, v)\right| d s d t+o(\log m \log n)
$$

and the lemma is proved.

\section{LEMMA 3.7 .}

$$
\begin{aligned}
& I_{2}=(1 / \pi) \log n \int_{1}^{m^{\frac{1}{2}}}\left|\int_{0}^{1} \frac{\operatorname{sinsu}}{s}\left\{d g(u, 1)-d g\left(u, 1^{-}\right)\right\}\right| d s+o(\log m \log n), \\
& I_{3}=(1 / \pi) \log m \int_{1}^{n^{\frac{1}{2}}}\left|\int_{0}^{1} \frac{\sin t v}{t}\left\{d g(1, v)-d g\left(1^{-}, v\right)\right\}\right| d t+o(\log m \log n) .
\end{aligned}
$$

Proof. We will prove the first part of the lemma. The second part follows in a similar manner.

Proceeding as in the proof of Lemma 3.1 with $\rho_{1}^{m}=1+\phi_{m}(s, u)$ and $\left|\phi_{m}(s, u) / s\right|<m s / 4$,

(3.8) $I_{2}=\int_{n^{-\frac{1}{2}}, 0}^{\pi, m^{-\frac{1}{2}}}\left|\int_{0,0}^{1,1} \rho_{1}^{m} \rho_{2}^{n} \frac{\operatorname{sinmsu}}{s} \frac{\sin n t v}{t} d g(u, v)\right| d s d t$

$$
=\int_{n^{-\frac{1}{2}}, 0}^{\pi, m^{-\frac{1}{2}}}\left|\int_{0,0}^{1,1} \rho_{2}^{n} \frac{\sin m s u}{s} \frac{\sin n t v}{t} d g(u, v)\right| d s d t+o(\log m \log n)
$$

since 


$$
\begin{aligned}
\int_{n^{-\frac{1}{2}}, 0}^{\pi, m^{-\frac{1}{2}}} \mid \int_{0,0}^{1,1} \rho_{2}^{n} \phi_{m}(s, u) \frac{\operatorname{sinmsu}}{s} \frac{\sin n t v}{t} & d g(u, v) \mid d s d t \\
& <\int_{n^{-\frac{1}{2}}, 0}^{\pi, m^{-\frac{1}{2}}}(m s) /(4 t) \int_{0,0}^{1,1}|d g(u, v)| d s d t \\
& =(1 / 8) v(g) \log \pi n^{\frac{1}{2}} \\
& =0(\log m \log n) .
\end{aligned}
$$

Next set

$$
\begin{aligned}
& \int_{0,0}^{1,1} \rho_{2}^{n} \sin m s u \sin n t v d g(u, v) \\
& \quad=\left\{\int_{0,0}^{0^{+}, 1}+\int_{0^{+}, 0}^{1^{-}, 1}+\int_{1-, 0}^{1,1}\right\} \rho_{2}^{n} \sin m s u \sin n t v d g(u, v) \\
& =i_{1}+i_{2}+i_{3} .
\end{aligned}
$$

Since $g(u, v)$ is a regular weight function, $g(u, 0)=g\left(u, 0^{+}\right)$, and so

$$
\left|i_{1}\right| \leq \int_{0,0}^{0^{+}, 1}|d g(u, v)|=\int_{0}^{1}\left|d g\left(u, 0^{+}\right)-d g(u, 0)\right|=0 .
$$

To estimate $i_{2}$, note that here $0<t \leq \pi$ and $0<v<1$ so that, by $(2.6)$,

$$
\rho_{2}^{2}<e^{-2 v(1-v) t^{2} / \pi^{2}}
$$

Hence

$$
\rho_{2}^{n}<e^{-n v(1-v) t^{2} / \pi^{2}}
$$

and so $\rho_{2}^{n}$ tends to zero boundedly as $n+\infty$. Hence

$$
\begin{aligned}
i_{2} & =\int_{0^{+}, 0}^{1^{-}, 1} \rho_{2}^{n} \sin m s u \sin n t v d g(u, v) \\
& =o(1) \int_{0^{+}, 0}^{1^{-}, 1} \sin m s u \sin n t v d g(u, v) .
\end{aligned}
$$


Finally,

$$
\begin{aligned}
i_{3} & =\int_{1^{-}, 0}^{1,1} \rho_{2}^{n} \sin m s u \sin n t v d g(u, v) \\
& =\int_{0}^{1} \sin m s u \sin n t\left\{d g(u, 1)-d\left(u, 1^{-}\right)\right\}
\end{aligned}
$$

since $\rho_{2}^{n}=1$ when $v=1$. Collecting these estimates and replacing $m s$ and $n t$ by $s$ and $t$ respectively, it follows that

(3.9) $I_{2}=\int_{n^{\frac{1}{2}}, 0}^{\pi n, m^{\frac{1}{2}}}\left|i_{1}+i_{2}+i_{3}\right| \frac{d s}{s} \frac{d t}{t}$

$$
\begin{aligned}
& =\int_{n^{\frac{1}{2}}, 0}^{\pi n, m^{\frac{1}{2}}}\left|\int_{0}^{1} \frac{\operatorname{sinsu}}{s} \frac{\sin t}{t}\left\{d g(u, 1)-d g\left(u, 1^{-}\right)\right\}\right| d s d t \\
& +\int_{n^{\frac{1}{2}}, 0}^{\pi n, m^{\frac{1}{2}}}\left\{o(1) \int_{0^{+}, 0}^{1^{-}, 1} \frac{\operatorname{sinsu}}{s} \frac{\sin t v}{t} d g(u, v)\right\} d s d t \\
& =\int_{n^{\frac{1}{2}}, 0}^{\pi n, m^{\frac{1}{2}}}\left|\int_{0}^{1} \frac{\operatorname{sinsu}}{s} \frac{\sin t}{t}\left\{d g(u, 1)-d g\left(u, 1^{-}\right)\right\}\right| d s d t \\
& +o(\log m \log n)
\end{aligned}
$$

since

$$
\begin{aligned}
& \int_{n^{\frac{1}{2}}, 0}^{\pi n, m^{\frac{1}{2}}}\left\{o(1) \int_{0^{+}, 0}^{1^{-}, 1} \frac{\operatorname{sinsu}}{s} \frac{\sin t v}{t} d g(u, v)\right\} d s d t \\
& \leq o(1) \int_{n^{\frac{1}{2}}}^{\pi n} \frac{d t}{t}\left\{\int_{0}^{1} d s+\int_{1}^{m^{\frac{1}{2}}} \frac{d s}{s}\right\} \int_{0^{+}, 0}^{1^{-}, 1}|d g(u, v)| \\
& =o(1) \log \pi n^{\frac{1}{2}}\left(1+\frac{1}{2} \log m\right) V(g) \\
& =o(\log m \log n),
\end{aligned}
$$

where we have used the observation that for $0<s \leq 1,|(\sin s u) / s| \leq 1$ and for $1 \leq s, t,|(\sin s u) / s|<1 / s$ and $|(\sin t v) / t|<1 / t$.

Returning to the last integral in (3.9), note that 


$$
\int_{1}^{\infty}\left\{|\sin t|-\frac{2}{\pi}\right\} \frac{d t}{t}
$$

converges, and so

(3.10)

$$
\begin{aligned}
& \int_{n^{\frac{1}{2}}, 0}^{\pi n, m^{\frac{1}{2}}}\left|\int_{0}^{1} \frac{\operatorname{sinsu}}{s} \frac{\sin t}{t}\left\{d g(u, 1)-d g\left(u, 1^{-}\right)\right\}\right| d s d t \\
& \quad=\int_{n^{\frac{1}{2}}}^{\pi n}\{\{|\sin t|-(2 / \pi)\}+(2 / \pi)\} \frac{d t}{t} \int_{0}^{m^{\frac{1}{2}}}\left|\int_{0}^{1} \frac{\operatorname{sinsu}}{s}\left\{d g(u, 1)-d g\left(u, 1^{-}\right)\right\}\right| d s \\
& \quad=\{o(1)+(2 / \pi) \log \pi+(1 / \pi) \log n\} \int_{0}^{m^{\frac{1}{2}}}\left|\int_{0}^{1} \frac{\operatorname{sinsu}}{s}\left\{d g(u, 1)-d g\left(u, 1^{-}\right)\right\}\right| d s \\
& =(1 / \pi) \log n \int_{0}^{m^{\frac{1}{2}}}\left|\int_{0}^{1} \frac{\operatorname{sinsu}}{s}\left\{d g(u, 1)-d g\left(u, 1^{-}\right)\right\}\right| d s+o(\log m \log n)
\end{aligned}
$$

since

$$
\int_{0}^{m^{\frac{1}{2}}}\left|\int_{0}^{1} \frac{\operatorname{sinsu}}{s}\left\{d g(u, 1)-d g\left(u, 1^{-}\right)\right\}\right| d s=o(\log m)=o(\log m \log n) .
$$

Finally, shifting the lower limit of integration with respect to $s$ from 0 to 1 in the last integral in (3.10) introduces an error of $(1 / \pi) \log n \int_{0}^{1}\left|\int_{0}^{1} \frac{\sin s u}{s}\left\{d g(u, 1)-d g\left(u, 1^{-}\right)\right\}\right| d s$

$$
\begin{aligned}
& <(1 / \pi) \log n \int_{0}^{1} d s \int_{0}^{1}\left|d g(u, 1)-d g\left(u, 1^{-}\right)\right| \\
& \leq(1 / \pi) V(g) \log n \\
& =o(\log m \log n) .
\end{aligned}
$$

Hence

$$
I_{2}=(1 / \pi) \log n \int_{1}^{m^{\frac{1}{2}}}\left|\int_{0}^{1} \frac{\operatorname{sinsu}}{s}\left\{d g(u, 1)-d g\left(u, 1^{-}\right)\right\}\right| d s+o(\log m \log n)
$$

and the lemma is proved. 
LEMMA 3.11.

$$
I_{4}=\left(1 / \pi^{2}\right) \lg \left(1,1 ; 1^{-}, 1^{-}\right) \mid \log m \log n+o(\log m \log n) .
$$

Proof. We have

$$
I_{4}=\int_{n^{-\frac{1}{2}}, m^{-\frac{1}{2}}}^{\pi, \pi}\left|\int_{0,0}^{1,1} \rho_{1}^{m} \rho_{2}^{n} \frac{\operatorname{sinmsu}}{s} \frac{\sin n t v}{t} d g(u, v)\right| d s d t .
$$

As in the proof of Lemma 3.7 , let

$$
\begin{aligned}
\int_{0,0}^{1,1} \rho_{1}^{m} \rho_{2}^{n} \sin & m s u \sin n \operatorname{tvdg}(u, v) \\
& =\left\{\int_{0,0}^{0^{+}, 1}+\int_{0^{+}, 0}^{1^{-}, 1}+\int_{1^{-}, 0}^{1,0^{+}}+\int_{1^{-}, 1}^{1,1}\right\} \rho_{1}^{m} \rho_{2}^{n} \sin m s u \sin n t v d g(u, v) \\
& =i_{1}+i_{2}+i_{3}+i_{4}+i_{5} .
\end{aligned}
$$

Since $g\left(u, 0^{+}\right)=g(u, 0)$,

$$
\left|i_{1}\right| \leq \int_{0,0}^{0^{+}, 1}|d g(u, v)|=\int_{0}^{1}\left|d g\left(u, 0^{+}\right)-d g(u, 1)\right|=0 .
$$

By the same argument, $i_{3}=0$.

To estimate $i_{2}$, for $t>0$ and $0<v<1, p_{2}^{n} \rightarrow 0$, and so $\rho_{1}^{m} \rho_{2}^{n} \sin m s u \sin n t v \rightarrow 0$ boundedly as $n \rightarrow \infty$. Hence

$$
\begin{aligned}
i_{2} & =\int_{0^{+}, 0}^{1^{-}, 1} \rho_{1}^{m} \rho_{2}^{n} \sin m s u \sin n t v d g(u, v) \\
& =o(1) .
\end{aligned}
$$

By a similar argument, $i_{4}=o(1)$. Finally,

$$
\begin{aligned}
i_{5} & =\int_{1^{-}, I^{-}}^{1,1} \rho_{1}^{m} \rho_{2}^{n} \sin m s u \sin n t v d g(u, v) \\
& =g\left(1,1 ; 1^{-}, 1^{-}\right) \sin m s \sin n t .
\end{aligned}
$$

Collecting the results, 
(3.12) $I_{4}=\int_{n^{-\frac{1}{2}}, m^{-\frac{1}{2}}}^{\pi, \pi}\left|i_{1}+i_{2}+i_{3}+i_{4}+i_{5}\right| \frac{d s}{s} \frac{d t}{t}$

$$
\begin{aligned}
& =\left|g\left(1,1 ; 1^{-}, 1^{-}\right)\right| \int_{n^{-\frac{1}{2}}, m^{-\frac{1}{2}}}^{\pi, \pi}\left|\frac{\operatorname{sinms}}{s} \frac{\sin n t}{t}\right| d s d t \\
& +\int_{n^{-\frac{1}{2}}, m^{-\frac{3}{2}}}^{\pi, \pi} o(1) \frac{d s}{s} \frac{d t}{t} \\
& =\left|g\left(1,1 ; 1^{-}, 1^{-}\right)\right| \int_{n^{\frac{1}{2}}, m^{\frac{1}{2}}}^{\pi n, \pi m}\left|\frac{\sin s}{s} \frac{\sin t}{t}\right| d s d t+o(\log m \log n) .
\end{aligned}
$$

Next, as in $(3.10)$,

$$
\int_{n^{\frac{1}{2}}}^{\pi n}\left|\frac{\sin t}{t}\right| d t=O(1)+(1 / \pi) \log n
$$

and similarly

$$
\int_{m^{\frac{1}{2}}}^{\pi m}\left|\frac{\sin s}{s}\right| d s=O(1)+(1 / \pi) \log m \text {. }
$$

Applying these estimates to (3.12), we get

$I_{4}=\lg \left(1,1 ; 1^{-}, 1^{-}\right) \mid\{O(1)+(1 / \pi) \log m\}\{O(1)+(1 / \pi) \log n\}+o(\log m \log n)$

$=\left(1 / \pi^{2}\right) \lg \left(1,1 ; 1^{-}, 1^{-}\right) \mid \log m \log n$

$+O(1)+O(\log m)+O(\log n)+O(\log m \log n)$

$=\left(1 / \pi^{2}\right) \lg \left(1,1 ; 1^{-}, 1^{-}\right) \mid \log m \log n+o(\log m \log n)$

and the lemma is proved.

To complete the proof of Theorem 1.3, we collect the results of Lemmas $3.1,3.7$ and 3.11 , and get, by Theorem 2.11, 


$$
\begin{aligned}
\left(\pi^{2} / 4\right) L(m, n ; g)= & \int_{0,0}^{\pi, \pi}\left|\int_{0,0}^{1,1} \rho_{1}^{m} \rho_{2}^{n} \frac{\operatorname{sinmsu}}{s} \frac{\sin n t v}{t} d g(u, v)\right| d s d t \\
& =\int_{1,1}^{n, m}\left|\int_{0,0}^{1,1} \frac{\operatorname{sinsu}}{s} \frac{\sin t v}{t} d g(u, v)\right| d s d t \\
& +(1 / \pi) \log m \int_{1}^{n}\left|\int_{0}^{1} \frac{\sin t v}{t}\left\{d g(1, v)-d g\left(1^{-}, v\right)\right\}\right| d t \\
& +(1 / \pi) \log n \int_{1}^{m}\left|\int_{0}^{1} \frac{\operatorname{sinsu}}{s}\left\{d g(u, 1)-d g\left(u, 1^{-}\right)\right\}\right| d s \\
& +\left(1 / \pi^{2}\right) \lg \left(1,1 ; 1^{-}, I^{-}\right) \mid \log m \log n+o(\log m \log n) .
\end{aligned}
$$

Multiplying both sides by $\left(4 / \pi^{2}\right)$ completes the proof of the main part of the theorem. The rest is obvious.

\section{References}

[1] C.R. Adams, "Hausdorff transformations for double sequences", BulZ. Amer. Math. Soc. 39 (1933), 303-312.

[2] C.R. Adams, "On definitions of bounded variation for functions of two variables", Trans. Amer. Math. Soc. 35 (1933), 824-854.

[3] A.E. Livingston, "The Lebesgue constants for Euler $(E, p)$ summation of Fourier series", Duke Math. J. 21 (1954), 309-314.

[4] L. Lorch and D.J. Newman, "The Lebesgue constants for regular Hausdorff methods", Canad. J. Math. 13 (1961), 283-298.

[5] 0. Szász, "Gibbs phenomenon for Hausdorff means", Trans. Amer. Math. Soc. $28(1926), 50-73$.

[6] F. Ustina, "Gibbs phenomenon for the Hausdorff means of double sequences", J. Austral. Math. Soc. 11 (1970), 169-185.

Department of Mathematics, University of Alberta,

Edmonton,

Canada T6G 261 . 